Recepción: 13 / 06 / 2018

Aceptación: 09 / 09 / 2018

Publicación: 05 / 10 / 2018

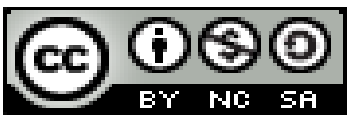

Ciencias de la educación

Artículo de investigación

\title{
La necesidad de la transdisciplinariedad en la educación
}

\author{
The need for transdisciplinarity in education
}

A necessidade da transdisciplinaridade na educação

\author{
Johnny P. Morquecho-Villalta ${ }^{\text {I }}$ \\ jhonypatrix@gmail.com
}

Correspondencia:_jhonypatrix@gmail.com

I Docente de la Universidad Nacional de Educación, Azogues, Ecuador. 


\title{
Resumen
}

El sistema educativo de la actualidad se encuentra en constantes cambios producidos por las sociedades modernistas, la globalización y las sociedades de información y el conocimiento, por lo que este artículo tiene como objetivo aportar un modelo de enseñanza-aprendizaje transdisciplinario que integre todos los conocimientos científicos y no científicos de la sociedad. El cual se genera a partir de la historia de vida de una docente y una estudiante de un centro educativo ecuatoriano, en donde se originó una confrontación de saberes ancestrales, con los conocimientos científicos y una falta de valores en una clase de Ciencias Naturales. Por lo que se presenta a la transdisciplinariedad como método idóneo para integrar la Educación antropológica social, la Educación en Valores y la Educación Ambiental; con el fin de explotar los talentos y capacidades de los individuos, desarrollar un perfeccionamiento en la personalidad humana y un pensamiento consiente y reflexivo con la naturaleza.

Palabras clave: transdisciplinariedad; antropología social y cultural; educación en valores; educación ambiental.

\begin{abstract}
Today's education system is constantly changing due to modern societies, globalization and information and knowledge societies, so this article aims to provide a transdisciplinary teachinglearning model that integrates all scientific and academic knowledge. Not scientists of society. Which is generated from the history of a life and a student of an Ecuadorian educational center, where a confrontation of ancestral knowledge, with technical knowledge and a lack of values in a Natural Sciences class. Transdisciplinarity presented as an ideal method to integrate social anthropological education, education in values and environmental education; In order to exploit the talents and abilities of individuals, develop a refinement in the human personality and a conscious and reflective thinking with nature.
\end{abstract}

Key words: transdisciplinarity; social and cultural anthropology; education in values; environmental education. 


\section{Resumo}

O sistema educacional de hoje está em constantes mudanças produzidas pelas sociedades modernistas, globalização e sociedades da informação e do conhecimento, portanto, este artigo tem como objetivo fornecer um modelo transdisciplinar de ensino-aprendizagem que integre todo o conhecimento científico. e não cientistas da sociedade. Que é gerado a partir da história de vida de um professor e um estudante de um centro educacional equatoriano, onde um confronto de conhecimentos ancestrais, com conhecimento científico e falta de valores em uma aula de Ciências Naturais. Portanto, a transdisciplinaridade é apresentada como um método ideal para integrar a educação antropológica social, a educação em valores e a educação ambiental; para explorar os talentos e habilidades dos indivíduos, desenvolva um refinamento na personalidade humana e uma abordagem ponderada e cuidadosa da natureza.

Palavras chave: transdisciplinaridade; antropologia social e cultural; educação em valores; Educação ambiental.

\section{Introducción}

Vivimos en un mundo cambiante, dinámico y globalizado que tiene como característica principal un crecimiento acelerado tanto en el ámbito de la información, el conocimiento, las comunicaciones y la tecnología; pero así también en el desarrollo industrial capitalista representado por el consumo masivo de bienes y servicios, que en general están desarrollando una crisis ecológica por la explotación irracional de los recursos materiales y energéticos de la naturaleza.

La escuela es una herramienta vital en todo este proceso dinámico de la sociedad actual, ya que es la encargada de formar a los individuos con las características intelectuales, cognitivas, espirituales, políticas y religiosas que la sociedad demanda. Y que hoy en día el conocimiento que se genera en las escuelas es a partir de disciplinas aisladas que no se combinan con los saberes ancestrales y el conocimiento científico.

Toda esta situación está generando nuevas demandas educativas con el objetivo de crear nuevos métodos y proceso de enseñanza que sean capaces de relacionar los contextos académicos con la naturaleza humana y su proceso evolutivo. (Moraes, 2007) Es por lo tanto la necesidad de una 
educación transdisciplinar que desarrolle seres continuamente concertados, capaces de adaptarse a las exigencias del mundo y que pueda potencializar las capacidades de cada individuo.

Consecuentemente esta educación transdisciplinar, debe combinar los saberes científicos, teóricos y metodológicos con los saberes de la antropología social, ya que según la Universidad Autónoma Indígena de México (2007) la antropología es una disciplina en donde el ser humano es un sujeto de estudio, al cual se investiga todos sus conocimientos empíricos, su organización, su diferenciación política y cultural, etc. Y por lo tanto su combinación con las diferentes disciplinas científicas y técnicas generan una multiplicidad de saberes, de las disciplinas científicas y de las tecnociencias (Moraes, 2007) desarrollado una interconexión entre los saberes bajo la consigna de un pensamiento expandido que ligue lo simple con lo complejo, lo micro con lo macro, lo singular con lo universal.

\section{Importancia del problema}

Esta investigación se realiza a partir de historias de vida de una docente y una estudianteح:

La experiencia de Curina, la niña kichwa amazónico en la asignatura de Ciencias Naturales, en el sexto grado de Educación General Básica de la Unidad Educativa Intercultural Bilingüe Amawta Samay, en Limoncoha, provincia de Sucumbíos.

\section{Historia de vida:}

Curina se sintió feliz, la profesora les llevó a la chakra del colegio, y nos preguntó si conocíamos algo de ella, o de los huertos familiares o comunitarios. Yo conocía por mis padres y abuelos de la chakra, y en el momento que iba a levantar la mano para responder, Carlos, uno de los alumnos mestizos del aula, empezó a responder, que su papá implementa en la comunidad la agricultura intensiva porque produce más productos para mantener el poder adquisitivo de su familia y que por eso priorizaba la producción con plaguicidas: insecticidas, herbicidas y fungicidas.

Antay y Libiak, dos de mis compañeros del aula de nacionalidad shuar, le pidieron permiso a la maestra para hablar y explicar que el papá de Carlos está provocando una intensa degradación de los valores del suelo, estimulando efectos negativos del medio ambiente. 
La profesora le pide a Curina que explique lo que ella conoce sobre el tema. Enseguida Curina explica que en un campo existen las plantas cultivadas. Y que también están las llamadas "malas hierbas", que acompañan a las plantas cultivadas, llamadas así por el agricultor cuando no son deseadas. Por ejemplo, los trigos de paja alta, limitan mucho el crecimiento de otras hierbas, sin necesidad de herbicidas. Por eso tenemos que aprender a elegir las "compañeras" de las plantas cultivadas y asociar estas especies, lo que permite el control de plantas no deseadas.

Estas plantas se llaman compañeras y dan lugar a asociaciones, por ejemplo, el maíz con leguminosa, o cultivo bajo de árboles. Incluso se pueden sembrar solo para facilitar el suelo y protegerlos sin utilizar químicos. $\mathrm{Y}$ es que las malas hierbas tienen usos alimentarios, medicinales y ornamentales, en ellas habitan muchos insectos, búhos, lechuzas, cernícalo, gorrión común, culebras, la lagartija común, la salamanquesa. Y como hay agua, también hay sapos, ranas y mariposas.

También aparecen hongos que se relacionan con las plantas por las raíces, por medio de algo que no recuerda el nombre. La profesora la ayuda y plantea que se llamaban micorrizas que protegen a las plantas de factores de estrés: como sequía, salinidad, contaminantes y enfermedades de raíz, por ello cada uno vive y crece con ayuda del otro. Mis abuelos siempre me dicen, que todos esos procedimientos que seguimos son para restablecer el equilibrio de la naturaleza. Por eso la tierra hay que cuidarla y respetarla, como mismo hemos con nuestra madre, ella será siempre parte de nuestra existencia.

Un estudiante mestizo llamado Luis, plantea que su papá es ingeniero químico, y el conoce que el avance de la agroquímica fue resultado de los avances científicos de finales del siglo XIX y del periodo de guerras, como una respuesta al hambre. En aquella época fue considerado un avance científico importante, esa idea no ha cambiado mayormente con el sistema agroindustrial que toma y aplica los avances científicos relacionados con el área. Y de otra parte hay un conjunto de conocimientos y prácticas de agricultura que implican relacionamientos otros entre seres humanos y con la naturaleza, que están presenten en los pueblos mestizos, indígenas, afro y montubios.

Antay, plantea que su abuelo le había explicado que la alfalfa es un cultivo de mucha tradición, y que ayuda mucho a las otras plantas que se están sembrando. La profesora explicó que la alfalfa 
es una leguminosa que capta el nitrógeno disuelto en el agua del suelo y fija el nitrógeno mediante la colaboración, que se le llama simbiosis, de una bacteria que vive en los nódulos de sus raíces (Sinorhizobium meliloti), y seguro era por eso que su abuelo la siembra junto a otros cultivos.

La profesora Magdalena, se había dado cuenta que había iniciado su trabajo en la escuela sin indagar y conocer a sus alumnos, el contexto en donde viven y sus experiencias. Por lo que reflexiona en cómo pudo haber dado la espalda ante tantos saberes, tecnologías, agro diversidad, y a toda una cultura que sigue vigente y resistiendo a pesar del impacto que genera la expansión urbana. Se da cuenta que tiene tantos alumnos con conocimientos sobre estos temas, pero que, sin embargo, no utiliza esas fortalezas para desarrollar una clase con metodologías innovadoras que favorezca no solo el conocimiento de los ecosistemas, sino de valores hacia el medioambiente.

Bajo este contexto de conocimientos científicos, teóricos y culturales se observa que en este problema la investigación tiene como objetivo orientar los procesos educativos hacia la práctica de la transdisciplinariedad visto como un método idóneo para promover las competencias profesionales al combinar los saberes científicos y no científicos de la sociedad, y a más de esto complementar dicho proceso con una Educación en Valores y una Educación Ambiental.

\section{Metodología}

La metodología que se utiliza para la investigación es el estudio de las historias de vida de una docente y una estudiante de la Unidad Educativa Intercultural Bilingüe "Amawta Samay" en el sexto año de Educación General Básica de Limoncocha, provincia de Sucumbíos del estado de Ecuador. En su grupo de 20 estudiantes: De ellos 7 mestizos (1 mujer y 6 varones), y 13 de nacionalidades indígenas ( 7 mujeres y 6 varones). Y de la niña

Consecuentemente se realiza el análisis de documentos con el fin de argumentar posibles soluciones al problema identificado.

\section{Resultados}

Según el estudio de las historias de vida analizado, se nota claramente la falta de investigación por parte de la docente a los estudiantes, ya que se necesitaba de una prueba de diagnóstico para conocer el nivel de conocimiento de los estudiantes y poder así, empezar su enseñanza

\section{4}

Pol. Con. (Edición núm. 26) Vol. 3, No 10, octubre 2018, pp. 19-31, ISSN: 2550 - 682 X 
aprendizaje partiendo del conocimiento previo de sus alumnos y desarrollar aprendizajes significativos.

Por otra parte el conocimiento del entorno natural y social en el que se encuentra la institución permite que el estudiante adquiera diferentes conocimientos que se generan a partir de la convivencia con su entorno, dando lugar a las experiencias previas en los estudiantes; mismo que fueron desaprovechados por la docente en el momento de la clase ya que la misma no aportó ningún conocimiento empírico ni científico, llegando así a ser solamente un mediador en su práctica y no llegar a ninguna conclusión.

Por tal motivo la clase no tuvo una retroalimentación adecuada, debido a que los conocimientos empíricos y científicos de los estudiantes solo se expusieron en el debate y que ninguna de las concepciones de los estudiantes fue aceptadas o validadas porque lamentablemente la docente no pudo aportar de manera constructiva al mismo, es decir que los conocimientos solo quedaron como ideas divagantes.

\section{Discusión}

El sistema educativo de la actualidad se encuentra en constantes cambios, retos y desafíos propuestos por la sociedad, ya que desde el siglo XIX al siglo XX se ha generado más conocimientos y ciencias que en todos los años de la humanidad. El conocimiento científico desarrollado en este periodo originó la pulverización de la ciencia en una inmensidad de disciplinas cada vez más especializadas, utilizadas por cada comunidad de científicos según su área y temáticas de conocimiento. (Collado, Paradigmas Epistemológicos en Filosofía, Ciencia y Educación, 2016) generando así la fragmentación del saber y la separación de las disciplinas.

Hoy en día se necesita de una educación que cambie todos los estereotipos y conflictos creados por la sociedad; porque como se pudo observar en el problema de investigación, los saberes antropológicos de los individuos son conocimientos válidos y verificables, que puestos a un proceso de análisis permiten mejorar la autoformación de las condiciones humanas.

Según Collado \& Madroñero (2016) es primordial repensar de forma transdisciplinar los procesos de formación humana, con el objetivo de identificar y desarrollar las competencias necesarias 
para afrontar los retos de la globalización actual. Por lo que, el método transdisciplinar busca combinar los saberes científicos con los saberes antropológicos de la sociedad.

Por lo tanto, la transdisciplinariedad debe estar ligada a un saber antropológico, en donde se considere los conocimientos ancestrales, empíricos y culturales en la formación continua del individuo, por lo cual es necesario de:

\section{Una educación transdisciplinar con los saberes antropológicos y culturales}

La antropología educativa o antropología educacional tiene sus principios desde Platón hasta la época contemporánea cuya comprensión permite vislumbrar el contexto histórico en el que se eleva el conocimiento del ser humano; y que, relacionadas con conocimientos económicos, matemáticos, jurídicos, filosófico, culturales, etc. Permiten la integración del conocimiento de forma completa y no singular. (Martínez, 2006)

Según Antonio Colom, (1980) La antropología cultural acoge los fenómenos culturales en aportes simbólicos producidos por la interacción social que condicionan y determinan el comportamiento humano. Sin embargo, la educación no puede estar separada de los saberes culturales porque en definitiva ambos se construyen desde su interacción en un mismo contexto y los mismos protagonistas. Desde una visión intrínseca se puede decir que la educación depende de la cultura y que de la misma manera su innovación depende de la educación.

De este modo surge la necesidad de integrar los conocimientos por el método de la transdisciplinariedad, interrelacionado los saberes científicos con las necesidades técnicas y conocimientos antropológicos culturales, con el fin de dirigir a la condición humana propia y ajena en orden para obtener resultados productivos y prácticos en la sociedad. Sin embargo, la antropología no solo incide en el conocimiento; sino también, en el proceso de enseñanza aprendizaje, en la conducta, costumbres, socialización del individuo, formas de vida, ambiente cultural, etc. (Colom, 1980) Entonces la transdisciplinariedad combinado con los saberes antropológicos y culturales buscan educar al ser humano de acuerdo con su naturaleza física, cultural y su contexto social permitiéndole su perfeccionamiento en un sentido humanista.

Bajo este concepto el individuo adquiere un conocimiento complejo y divergente producto de los saberes científicos y no científicos, por lo que surge la necesidad de: 


\section{Una educación en valores ligada a la transdisciplinariedad}

Los cambios sociales y culturales originados por la globalización, revolución científica y tecnológica han promovido una crisis de valores y de los sistemas de creencias de la sociedad actual. (Parra, 2003). Así como la sociedad cambia el sistema de valores también se modifica; como, por ejemplo, para los tradicionalistas de orientación objetiva los valores que deben predominar en la sociedad son los "valores absolutos" (la verdad, el valor, la justicia, la equidad la libertad, la belleza y la bondad) pero para los modernistas el sistema de valores radica en la formación del individuo para enfrentar los problemas de la sociedad moderna, el aprovechamiento de las oportunidades sociales y de tener objetivos y metas individuales.

Según José Parra (2003) cada sociedad en un tiempo determinado, selecciona el conjunto de valores más adecuados para satisfacer las necesidades sociales, siendo la escuela la encargada de transmitir y desarrollar por medio de la actividad educativa. Dicha transmisión permite la formación de la personalidad de cada individuo mediante los procesos de enseñanza y aprendizaje con el objetivo de fomentar las interrelaciones con sus semejantes dentro de un contexto social.

Entre todo este preámbulo se produce la enseñanza de valores como una necesidad educativa, la cual es definida por Paniagua (2009) como el nuevo término que se emplea dentro de la educación cuando se trata de dificultades o problemas que presenta un alumno directamente relacionados con la adquisición de competencias académicas. Por lo tanto, se plantea la utilización de la transdisciplinariedad para la formación de una educación en valores; ya que los saberes antropológicos y culturales que se encuentran inmersos en este método contribuyen de manera significativa en la formación de la personalidad de cada individuo.

De este modo se busca que se integre el conocimiento con el sistema de valores en un método universal transdisciplinar que busque generar un pensamiento moral, ético y humanista, con la sociedad actual y un desarrollo sostenible respetando los recursos del planeta; por lo que la transdisciplinariedad también debe estar ligada a una educación ambiental. 


\section{La transdisciplinariedad integrada con la educación ambiental}

Dentro de la sociedad actual, el medio ambiente juega un papel secundario en el desarrollo del mismo, porque no se da la debida importancia para su cuidado y su conservación, por lo que se requiere que en la educación desde los grados inferiores hasta los superiores se brinden los conocimientos y bases necesarias para la conservación del entorno natural que nos rodea.

Previamente es necesario conocer ¿Que es la educación ambiental? Es una educación que proporciona una relación de las acciones de los seres humanos con su medio biofísico y cultural, que tiene como principal propósito crear una consciencia individual y colectiva referente a la conservación y cuidado del medio ambiente. En donde se implemente estrategias dinámicas de innovación y cambio en los procesos ambientales, culturales, económicos, sociales, entre otros; dirigidos a proteger la utilización racional de los recursos naturales. De ahí que la educación ambiental contribuye favorablemente a la construcción de una sociedad cultural para la sustentabilidad. (Barbosa, 2014)

La UNESCO (1992), define a la educación ambiental como un proceso en donde se reconoce los valores y se aclara conceptos con el objetivo de fomentar aptitudes y actitudes necesarias para comprender la relación existente entre el hombre, su cultura y el ambiente. Comprende también la práctica en la toma de decisiones y en la elaboración de un código de comportamiento con respecto a las cuestione ambientales. (Reyes, 2009)

Se entiende como educación ambiental al proceso que incluye normas, reglas, valores y acciones direccionadas al cuidado y conservación del medio ambiente, la misma que el ser humana la realiza a través de estrategias y tomas de decisiones enfocadas a proteger el entorno natural. También se enfoca en la relación bilateral existente entre el ser humano, la cultura y el ambiente.

Cabe recalcar que para la autora Reyes (2009) la educación ambiental busca ayudar a la persona y a los grupos sociales a que adquieran mayor sustentabilidad y conciencia del medio ambiente. Por lo que presenta los siguientes objetivos presentes en la educación ambiental:

Desarrollar capacidades de análisis, reflexión y acción individual y colectiva.

Resolver problemas

Concientizar 
Cambiar hábitos

Cambiar actitudes

Cambiar comportamientos y valores

La transdisciplinariedad es el medio para alcanzar dichos objetivos. Se determinó que, para ser eficaz, la educación en materia de medio ambiente y desarrollo debe ocuparse de la dinámica del medio físico y biológico, del medio socioeconómico y del ser humano, debe integrarse a todas las disciplinas y utilizar métodos académicos y no académicos, además de medios efectivos de comunicación. (Reyes, 2009) Se trata de la construcción de un sistema total que no tuviera fronteras sólidas entre las disciplinas. Entra en un plano de confluencia, articulación reticular y contextualización del conocimiento de un modo más pertinente, donde se establece la interrelación e interconexión entre las partes y el todo. (Delgado, 2009)

El enfoque transdisciplinario en la enseñanza del medio ambiente supone una mayor comunicación entre las ciencias sociales y naturales; se pretende unificar un conocimiento en relación con todas las materias, de manera sistémica. Es así que se busca consolidar los conocimientos desde el nivel de primaria hasta la universidad, para formar una conciencia ecológica de preservación del medio ambiente. (Pedroza \& Argüello, 2002)

La educación ambiental debe tener como base fundamental el método transdisciplinario para crear en cada uno de sus estudiantes una visión global, crítica y conservadora dentro de la ecología y preservación del medio ambiente. Por lo que se recomienda emplear este método desde la educación primaria hasta la superior, creando así dentro de la sociedad una conciencia ecológica, sólida, responsable y perdurable sobre la importancia del entorno que nos rodea y sus elementos.

\section{Conclusiones}

El sistema educativo debe modificarse porque las nuevas generaciones necesitan de una enseñanza-aprendizaje que contemple el conocimiento de la biodiversidad, condición y la conciencia humana, cuyo proceso se genera a partir de la integración de los saberes antropológicos y culturales de las sociedades en general, con el fin de explotar los talentos y capacidades humanas. Que complementado con una educación en valores que permita desarrollar 
un perfeccionamiento en la personalidad al ser competente ante las problemáticas que se le presentan y aprovechar las oportunidades sociales para cumplir los objetivos y metas de la sociedad en el que se encuentra inmerso.

Por otra parte, la integración de la educación ambiental con la transdisciplinariedad es un factor muy importante en desarrollo humano, porque permite al individuo conocer las causas y consecuencias de la destrucción de los recursos materiales y energéticos del planeta provocados por las sociedades pasadas y actuales; tratando así, de generar pensamientos conscientes, críticos, reflexivos, creativos e innovadores con el medio natural que nos rodea.

\section{Referencias Bibliográficas}

Barbosa, A. (2014). HYPATIA: Revista de Divulgación Científico-Tecnológica del Gobierno del Estado de Morelos. Recuperado de: https://revistahypatia.org/educacion-amb-revista-34.html

Collado, J. (2016). Paradigmas Epistemológicos en Filosofía, Ciencia y Educación. En J. Collado, Paradigmas Epistemológicos en Filosofía, Ciencia y Educación. p. 21. España: Editorial Académica Española. Recuperado de: https://e.edim.co/16537772/gW1cURaf7lhxKQTZ.pdf?response-content-disposition=filename\%3 D\%22Paradigmas_epistemologicos_en_filosofia_ciencia_y_educacion_ensayos_cosmodernos.pd f\%22\%3B\%20filename $\% 2$ A\%3DUTF-8\%27\%27Paradigmas-epistemologicos-en-filosofiaciencia

Collado, J., \& Madroñero, M. (2016). Educación transdisciplinar: formando en competencias para el Buen Vivir. Recuperado de: https://e.edim.co/16537772/bGObbEyrXAoStwpK.pdf?response-content-disposition =filename\%3D\%22Educacio_n_transdisciplinar_formando_en_competencias_para_el_Buen_Vi vir.pdf

Colom, A. (1980). Antropología y Educación. Salamanca: Ediciones Universidad de Salamanca.

Delgado, R. (2009). La integración de los saberes bajo el enfoque dialéctico globalizador: La interdisciplinariedad y transdisciplinariedad en educación. Investigación y Postgrado, 24(3). 
Martínez, A. (2006). Antropología de la Educación para la formación de profesores (Vol. 9).

Educación y Educadores.

México, U. A. (2007). Antropología social. En U. A. México, Antropología social (pág. 497).

México: Mochicahui, el Fuente. Recuperado de:

http://uaim.edu.mx/carreras/etnopsicologia/05\%20trimestre/antropologia \%20social.pdf

Moraes, M. (2007). Complejidad, transdisciplinariedad y educación: Algunas reflexiones.

Recuperado de: http:/www.en cuentros-

multidisciplinares.org/Revistan\%C2\%BA25/Mar\%C3\%ADa\%20C\%C3\%A1ndida\%20Mora

les.pdf

Paniagua, M. (2009). Capítulo 9: Las Necesidades Educativas. Recuperado de: http://www.mailxmail.com/curso-integracion-escolar-social-nee-1-proceso-integracion-

escolar/necesidades-educativas-definicion

Parra, J. M. (2003). La Educación en Valores y su práctica en el Aula. Tendencias Pedagógicas, 70.

Pedroza, R., \& Argüello, F. (2002). Interdisciplinariedad y Transdisciplinariedad en los Modelos de Enseñanza de la Cuestión Ambiental. Cinta de Moebio: Revista de Epistemología de Ciencias Sociales, pp. 294-297.

Reyes, R. (2009). Por una educación ambiental. Visión docente con-ciencia, VIII (49), pp. 32-34. 